\title{
Development of a Nude Mouse Model to Study Human Sebaceous Gland Physiology and Pathophysiology
}

Marta J. Petersen, John J. Zone, and Gerald G. Krueger Division of Dermatology, Department of Internal Medicine, University of Utah Medical Center, Salt Lake City, Utah 84132

bstract. Study of human sebaceous gland physiology and pathophysiology is limited by lack of an adequate animal model. This study was designed to develop an animal model using human face skin grafted onto the nude mouse to study human sebaceous glands. Full-thickness human face skin was grafted onto 60 adult male nude mice. 4 wk after grafting, androgens, which are known to stimulate sebaceous glands, were administered to test the system. Androgens were administered to 21 animals by implanted catheters that were filled with testosterone (T) or dihydrotestosterone (DHT). Empty catheters were implanted in 15 control animals. Graft biopsies and blood for androgen levels were obtained at time 1 (pre-catheter) and time 2 (26 d after catheter implantation). Three assessments were made on each biopsy: sebaceous gland volume, using an image analyzing computer; sebaceous cell size; and sebaceous gland labeling index. 29 mice completed the study through time 2 . In the androgen-treated group, $T$ levels (nanogram per milliliter) five times increased to $4.92 \pm 0.35$, and DHT levels (nanogram per milliliter) increased 50 times to 16.70. In the androgen-treated group, sebaceous gland volume $\left(\mu \mathrm{m}^{3} \times 10^{-3}\right)$ increased from $896 \pm 194$ to $3,233 \pm 754(P<0.001)$, sebaceous cell area $\left(\mu \mathrm{m}^{2}\right)$ increased from $167 \pm 12$ to $243 \pm 19(P$ $<0.001$ ), and labeling index (percentage) increased from $2.7 \pm 0.7$ to $6.4 \pm 0.9(P<0.01)$. In the control group, sebaceous gland volume fell from $1,070 \pm 393$ to $417 \pm 99$

This work was presented at the National Meeting of the American Federation of Clinical Research in Washington, DC, 1984, and has been published in abstract form in Clin. Res., 1984, 32:257.

Dr. Petersen was the recipient of the 1983 Revlon Dermatology Foundation Research Fellowship. Address correspondence to Dr. Zone. 1984.

Received for publication 16 March 1984 and in revised form 7 June

J. Clin. Invest.

(C) The American Society for Clinical Investigation, Inc.

0021-9738/84/10/1358/08 \$1.00

Volume 74, October 1984, 1358-1365
(NS), sebaceous cell size remained the same, and the labeling index fell from $5.1 \pm 1.9$ to $3.2 \pm 1.1$. After androgen administration, Halowax N-34, a known comedogen, or its vehicle, was applied to 15 grafts for 2-6 wk. Twice as many microcomedones were seen in the Halowax-treated grafts, compared with vehicle-treated grafts at the end of this time period. No visible comedones were produced.

This study demonstrated that: $(a)$ human sebaceous glands can be successfully transplanted and studied on the nude mouse; $(b)$ after androgen stimulation, sebaceous gland volume, cell size, and labeling index increase; (c) microcomedones can be produced in the human skin grafts by the application of a comedogenic substance. Thus, this model demonstrates significant potential for the future study of human sebaceous gland physiology and pathology.

\section{Introduction}

Study of the physiology of the human pilosebaceous unit and diseases that affect it, notably acne vulgaris, has been hampered by the lack of an adequate animal model. Animal studies have focused on the investigation of rodent sebaceous glands and the pilosebaceous apparatus of the rabbit ear. However, significant differences have been found between the existing animal models and the data from human studies.

The effect of hormonal stimulation on sebaceous glands and sebum production has been studied extensively in the rat (1). Both the stimulatory effect of testosterone $(T)^{1}$ and the inhibitory effect of estrogen have been well documented with this model (1). The effects of adrenal androgens $(2,3)$ and the isolated effects of pituitary hormones on the sebaceous glands (4) have also been investigated. These data largely parallel human studies, but certain discrepancies have been found. Although progesterone has been shown by some investigators (5) to increase sebum production in rats, no similar effect has been documented in humans (6). In addition, correlative

1. Abbreviations used in this paper: DHT, $5 \alpha$-dihydrotestosterone; T, testosterone. 
human data are lacking on the effect of pituitary hormones in sebum production. Studies of androgen receptors within sebaceous cells have utilized rodent sebaceous gland analogues (7). Investigation of receptors in human sebaceous glands has been limited.

The external ear canal of the rabbit has been used to study comedogenicity of topically applied substances (8). However, the pilosebaceous units of the rabbit and man differ anatomically (9); there is a lack of reproducibility of results using this model, and comedones are more readily produced in response to topically applied substances in rabbit ear skin (10).

Secondary to its athymic state, the nude mouse is able to accept heterologous skin grafts (11). This system has been used extensively in the last decade to study epidermal cell kinetics in normal and diseased human skin (12). These studies have used split thickness skin grafts that lack intact sebaceous glands or other epidermal appendages. To date, transplantation of full-thickness human skin and long-term study of intact human sebaceous glands has not been attempted.

This study was designed to develop a model with which to study human sebaceous gland physiology and pathophysiology, using human skin grafted onto the nude mouse. We successfully grafted and maintained full-thickness human face skin on the nude mouse. We also demonstrated that the grafted human sebaceous glands became hyperplastic in response to exogenous androgen. After androgen administration, microcomedones were produced in the grafts by the application of a comedogenic substance.

\section{Methods}

Skin grafting. Face skin, remnants from the face-lift surgery of seven patients, was obtained in cooperation with local plastic surgeons. Age and an estimate of severity of adolescent acne were asked of each donor. The remnant skin was placed in phosphate-buffered saline (PBS) with $1 \%$ penicillin/streptomycin (Flow Laboratories, Inc., McClean, VA) during surgery and picked up by our personnel within $6 \mathrm{~h}$. The skin was keratomed to a depth of $0.88-0.92 \mathrm{~mm}$ with a Brown electrokeratome (Zimmer U.S.A., Inc., Warsaw, IN), placed in a sterile solution of RPMI-1640 (Flow Laboratories, Inc.) with $1 \%$ penicillin/streptomycin, and stored overnight at $4^{\circ} \mathrm{C}$.

Adult male CD-1 outbred nude mice that were obtained from our breeding colony were used, and housed and handled in a standard aseptic manner (13). 52 of the 60 animals that were grafted were castrated at least 1 wk before grafting in order to further control the endocrine milieu affecting the skin graft. In order to assess any potential effect of endogenous testosterone on the sebaceous glands, eight animals were not castrated. Retrospective evaluation of the noncastrated group at the end of the study failed to demonstrate any significant effect of endogenous testosterone. Consequently, the results of the castrated and the noncastrated groups were combined for subsequent data analysis.

Pre- and post-auricular, nonterminal hair-bearing skin samples, 1.5 $\times 1.5 \mathrm{~cm}$, were grafted orthotopically onto the left thoracic flank of the mice using previously discussed methods $(13,14)$. Eight mice (donors I-V) or 10 mice (donors VI and VII) were grafted with skin from each face-lift. Grafts were allowed to mature for 21-28 $d$ before catheters were implanted.
Microbiology. After overnight incubation of the keratomed skin, a $1-\mathrm{ml}$ sample of the incubating media was sent to the Microbiology Laboratory of this institution for aerobic and anaerobic culture. In addition, skin graft biopsies were obtained at 2 wk (one graft) and 12 wk (three grafts) post-grafting and cultured for anaerobes and aerobes. Androgen administration-catheter preparation. Testosterone and $5 \alpha$-dihydrotestosterone (DHT) catheters were prepared using the method described by DeMassa et al. (15) using Silastic medical grade tubing (Dow Corning Corp., Midland, MI) (internal diameter equals 0.191 $\mathrm{cm}$, outer diameter equals $0.316 \mathrm{~cm}$ ) and T or DHT crystals (Sigma Chemical Co., St. Louis, MO). The filled catheters were sterilized under ultraviolet $C$ light for $48 \mathrm{~h}$. Before implantation, they were incubated at $37^{\circ} \mathrm{C}$ for $48 \mathrm{~h}$ in sterile PBS $(1 \mathrm{ml} / 2$-cm catheter length) which was changed six times. Catheters were implanted subcutaneously via a $1-\mathrm{cm}$ incision on the dorsal flank of the animal opposite the skin graft.

Preliminary catheter study. A preliminary experiment to determine optimal $\mathrm{T}$ catheter length was performed using eight nongrafted castrated mice, and filled catheter lengths of 1,2 , and $3 \mathrm{~cm}$. T levels were measured at weeks $0,1,2,3,4,5,6,8$, and 10 . T levels were also measured in seven noncastrated mice.

Androgen administration to grafted animals. On the basis of the preliminary catheter study, 3-cm catheters (filled length) that contained T, DHT, or nothing (control catheters) were implanted in the skingrafted animals. 36 catheters were implanted: 19 T, 2 DHT, and 15 control. Testosterone and control catheters were implanted in an approximately equal number of mice from each donor group. Androgen levels were measured at time 1 (immediately before catheter implantation) and time 2 (a mean of $26 \mathrm{~d}$ after catheter implantation, range 9-28 d).

Testosterone and dihydrotestosterone levels. Approximately $0.1 \mathrm{ml}$ of blood was obtained at time 1 and time 2 using a retro-orbital bleed technique. The blood was centrifuged, the serum separated, and the sera was stored at $-70^{\circ} \mathrm{C}$ until analyzed. Total $\mathrm{T}$ levels were measured in all $\mathrm{T}$ treated and control animals using a Immunochem CovalentCoat Direct $\left(\mathbf{I}^{125}\right)$ Testosterone Radioimmunoassay Kit (Immuchem Corp., Carson, CA). DHT levels were assayed in the Clinical Research Laboratories of this institution (16)

Skin biopsies. 4-mm punch biopsies of donor grafts were obtained at time 1 and time 2. Each biopsy was divided in two equal parts. Half of the biopsy was fixed in formalin and sent for hematoxylin and eosin staining of serial sections. These sections were used to determine sebaceous gland volume and cell size. The other half of the biopsy was used for autoradiographic analysis of the sebaceous gland-labeling index.

Sebaceous gland volume. Sebaceous gland area was measured using an image-analyzing computer, the Quantimet 720 (Imanco, Monsey, NY), in conjunction with the Radiobiology Division, University of Utah, Salt Lake City. Sections were imaged under 100 and 250 times magnification (depending upon the size of the sebaceous gland), projected onto the video screen, and the perimeter of the sebaceous gland was traced with the light pen. The computer software calculated an area for each traced gland (17). All glands in every 20th section of the biopsy were outlined, for a total of seven sections per biopsy. A mean sebaceous gland area was calculated for each biopsy and an average sebaceous gland volume computed using the equation: volume $=(\text { mean area })^{3 / 2} \times 1.382(18)$.

The variability of sebaceous gland volume among multiple grafts from the same donor skin (intra-donor variability) was analyzed by measuring sebaceous gland volume and SEM of all surviving grafts 
from an individual donor at time 1 . Five donors had at least three surviving grafts at time 1 . The SEM for these five groups ranged from 261 to $682 \mu \mathrm{m}^{3} \times 10^{-3}$. It should be emphasized that in this study the variance introduced in comparing sebaceous gland volume from different donors and samples from the same donor was controlled by the fact that control and treated animals were paired with themselves at time 1 and time 2. This effectively eliminated the potential source of variance introduced by measurement of sebaceous gland volume between grafts.

Sebaceous cell area. One gland per biopsy was outlined, area obtained, and the number of cells in that gland counted. Nests of undifferentiated cells were not included in the area measurement or cell count. An average sebaceous cell area for one gland on each biopsy was calculated by dividing the gland area by the number of sebaceous cells in that gland. The intra-donor variability of sebaceous cells ranged from 11 to $36 \mu \mathrm{m}^{2}$ (SEM).

Labeling index. A 1-mm wide section of biopsy was incubated for $16 \mathrm{~h}$ at $37^{\circ} \mathrm{C}$ in a $10 \mathrm{ml}$ of RPMI-1640 (Flow Laboratories, Inc.) that contained $2 \mu \mathrm{Ci} / \mathrm{ml}$ of tritiated thymidine $(20.0 \mathrm{Ci} / \mathrm{mM})$ (New England Nuclear, Boston, MA). After incubation, the section was formalin fixed, and unstained semi-serial 6- $\mu \mathrm{m}$ sections were used for the autoradiography. Autoradiography was performed using Kodak NTB3 nuclear track emulsion (Eastman Kodak Co., Rochester, NY) and a 5-d exposure time (13). After development, the sections were counterstained with hematoxylin and eosin. The labeling index (number of labeled cells per unlabeled cells times 100 ) was calculated for time 1 and time 2. An average of 500 nonlipid-containing sebaceous cells were counted per biopsy. Sebaceous duct epithelial cells and undifferentiated cells adjacent to the duct were not counted. No distinction was made between the basal cells and nests of undifferentiated cells within the sebaceous gland fundus. Labeled cells had $\geq 6$ grains/cell; background labeling was $<10 \%$.

Halowax experiment. After time 2, a polychlorinated naphthalene compound and known comedogen (19), Halowax N-34 (obtained as a gift from Dr. Robert Cuozzo, Chemisphere Corp., Boonton, NJ), was applied to the human skin grafts in an attempt to produce follicular keratinization and microcomedo formation. An emulsion of mineral oil, acetone, and Halowax N-34 was prepared as described by Puhvel et al. (20). A 0.1-ml amount of this emulsion, which contained $20 \mathrm{mg}$ Halowax N-34, was applied $5 \mathrm{~d} / \mathrm{wk}$ for 10-30 applications onto 10 grafts (eight testosterone treated, two control). The vehicle, a mineral oil-acetone emulsion, was applied to the skin grafts on five animals (two testosterone treated, three control). Each graft was then removed and serially sectioned. The number of microcomedones present on $\sim 90$ sections/graft was assessed in a blinded fashion. Microcomedones present in multiple serial sections were tabulated only once. Presence or absence of microcomedones in the human skin grafts before Halowax application was also assessed.

Statistics. Descriptive statistics are represented as the mean \pm SEM. Change in sebaceous gland indices (volume, cell size, labeling index) in the androgen treated and control grafts was assessed using a paired $t$ test (21) which compared each graft to itself at time 1 and time 2 . The use of paired samples eliminated the variability of measurement between grafts. Data were also analyzed using the Wilcoxon signed rank test, a nonparametric test of paired samples (21), where the constraints of the $t$ test were not thought to be met.

\section{Results}

Face-lift skin donors. Face-lift skin from six females and one male donor was used. Six of the seven face-lift donors were between 53 and $61 \mathrm{yr}$ of age, and one was 37 . Adolescent acne history obtained from six of the seven donors was none in three, mild in two, and moderately severe in one donor.

Graft survival. A total of 60 animals were grafted with skin from seven face-lift donors. Fig. 1 illustrates a mouse with grafted human skin, 8 wk post-grafting. 29 animals completed the study through time 2 , and gave an overall completion rate of $48.3 \%$. These 29 animals were grafted from the following face-lift donor groups: group I, three animals; group II, one animal; group III, four animals, group IV, six animals; group V, one animal; group VI, eight animals; and group VII, six animals. Groups I-V, which were the first to be grafted, had a dropout rate of $50 \%(20 / 40)$ and was secondary to animal deaths (16/40) and graft death (4/40). Groups VI and VII were grafted at a later date with younger mice. The mortality rate before catheter implantation in these two groups was $20 \%$ (4/ 20), which was secondary to four animal deaths.

After implantation of the catheters in 36 animals, three animals died before biopsy, two animals were eliminated due to poor graft viability on biopsy, and two animals were eliminated due to an error in specimen handling. 24 of the 29 mice that completed the study were biopsied $28 \mathrm{~d}$ after catheter implantation (time 2). Five mice were biopsied earlier (days $9,11,11,18$, and 18) due to mouse morbidity.

Microbiology results. In no case did culture of the incubating skin broth yield anaerobes, or, more specifically, propionibacterium acnes or propionibacterium granulosum. A pseudomonas species, subsequently cultured from a water source, was cultured from the media from groups I-IV. A skin biopsy specimen that was obtained 2 wk post-grafting from an animal in group II showed no growth of the pseudomonas species. Skin culture of 3 12-wk-old grafts revealed no anaerobic or aerobic growth.

Preliminary catheter study. The mean T level (nanogram per milliliter) in seven noncastrated adult CD-1 male nude mice was $2.44 \pm 0.96$, with a range of $0.81-7.89$. The mean $T$

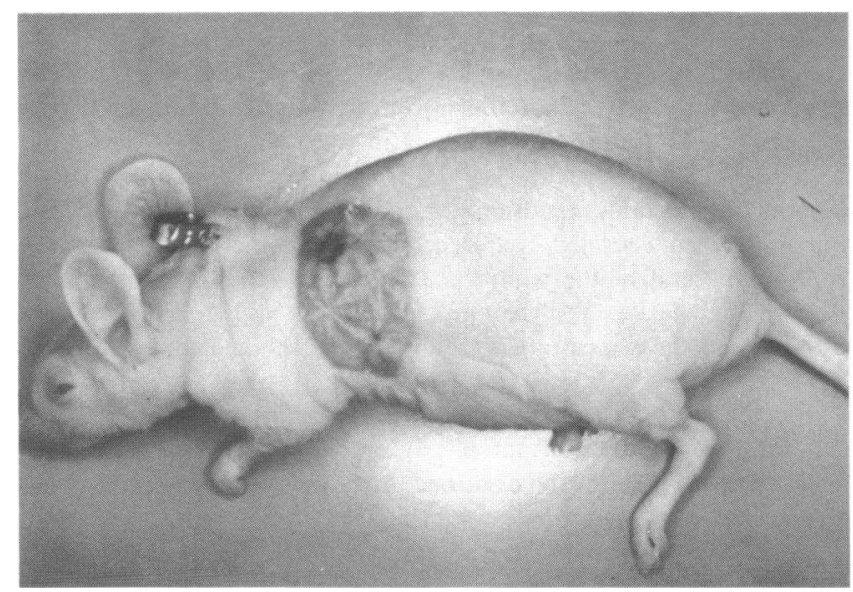

Figure 1. Athymic mouse with human skin graft, 8 wk post-grafting. 
Table I. T Levels in T Treated and Control Mice

\begin{tabular}{|c|c|c|c|c|}
\hline & \multicolumn{2}{|l|}{ Testosterone } & \multicolumn{2}{|l|}{ Control } \\
\hline & Time 1 & Time 2 & Time 1 & Time 2 \\
\hline \multicolumn{5}{|l|}{ Testosterone } \\
\hline$n$ & 16 & 17 & 9 & 10 \\
\hline
\end{tabular}

Data represent mean \pm SEM. Data from two samples were eliminated from the analysis at time 1 .

level in seven castrated adult CD- 1 nude mice before catheter implantation (week 0 ) was $0.57 \pm 0.21$, with a range of 0.09 1.44. 4 wk after catheter implantation, the following mean $T$ levels (nanogram per milliliter) were obtained: for a $1-\mathrm{cm}$ catheter, $4.57 \pm 0.14(n=2)$; for a 2 -cm catheter, $4.47 \pm 1.09(n$ $=3$ ); and for a 3-cm catheter, 7.85 $\pm 1.17(n=3)$. T levels at weeks 6,8 , and 10 in two mice with two 3 -cm catheters were $7.90 \pm 0.70,7.53 \pm 1.83$, and $5.16 \pm 1.07$, respectively. On the basis of these results, 3-cm catheters, filled with $T$ or DHT, were implanted into the grafted study mice.

A

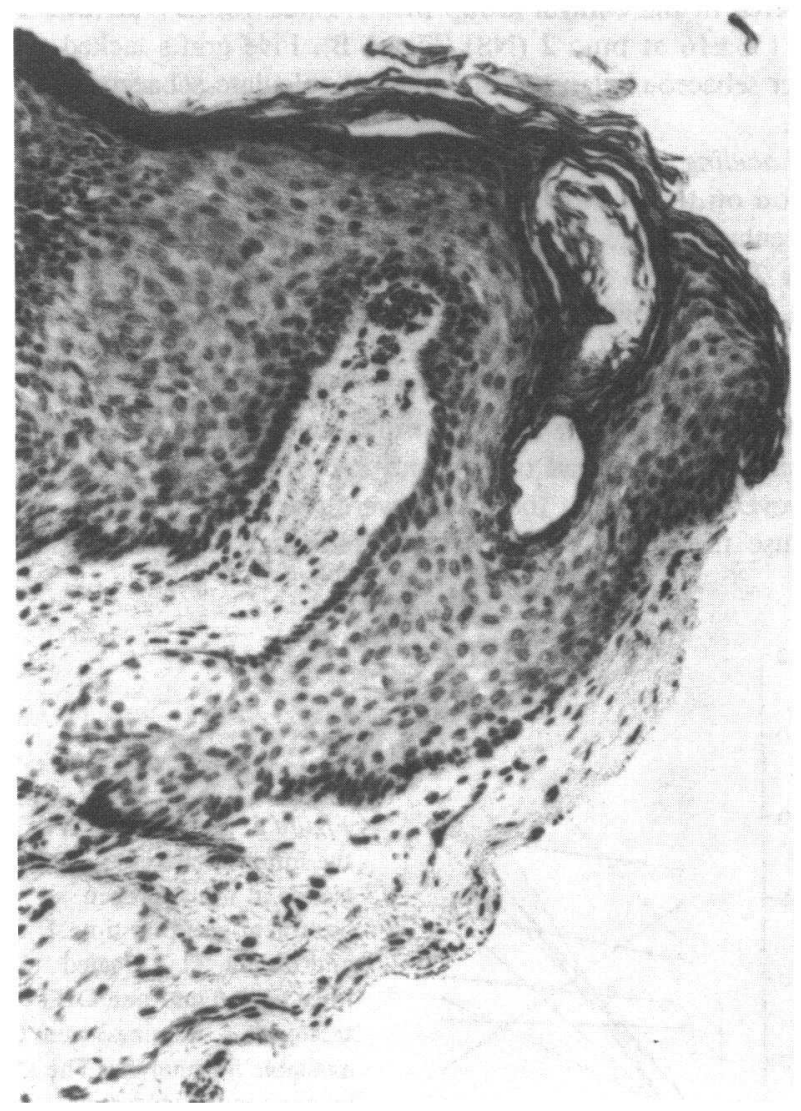

Figure 2. (A) Biopsy from human face skin graft $4 \mathrm{wk}$ after grafting. $\times 100$. (B) A repeat biopsy from the same graft after $28 \mathrm{~d}$ of
Androgen levels in the grafted animals. T levels (nanogram per milliliter) in the $T$ treated and control groups are shown in Table $I$. The $T$ levels in two animals at time 1 were eliminated due to a technical problem with the assay. It can be seen that the $T$ level increased fivefold in the $T$-treated group and remained relatively constant in the control group. DHT levels (nanogram per milliliter) in the two animals that were implanted with DHT catheters were $0.31 \pm 0.28$, precatheter, and 16.70 , a single value from a pooled specimen, $28 \mathrm{~d}$ post catheter implantation.

Sebaceous gland volume. Fig. 2 is a photomicrograph of a skin biopsy before (A) and $28 \mathrm{~d}$ after (B) implantation of a $\mathrm{T}$ filled catheter, which illustrates the qualitative increase in sebaceous gland size that is seen with $\mathrm{T}$ stimulation. Fig. $3 \mathrm{~A}$ shows the effect of T $(n=17)$ and DHT $(n=2)$ administration on sebaceous gland volume. The mean sebaceous gland volume increased $260 \%$ with androgen stimulation, from $896 \pm 194$ $\left(\mu \mathrm{m}^{3} \times 10^{-3}\right)$ at time 1 to $3,233 \pm 754$ at time 2 . A paired $t$ test, comparing each graft to itself at time 1 and time 2, was significant at the 0.001 level. Sebaceous gland volume decreased in four of the treated grafts, attendant with a low $T$ level in one. No sebaceous glands were seen in two of those four

B

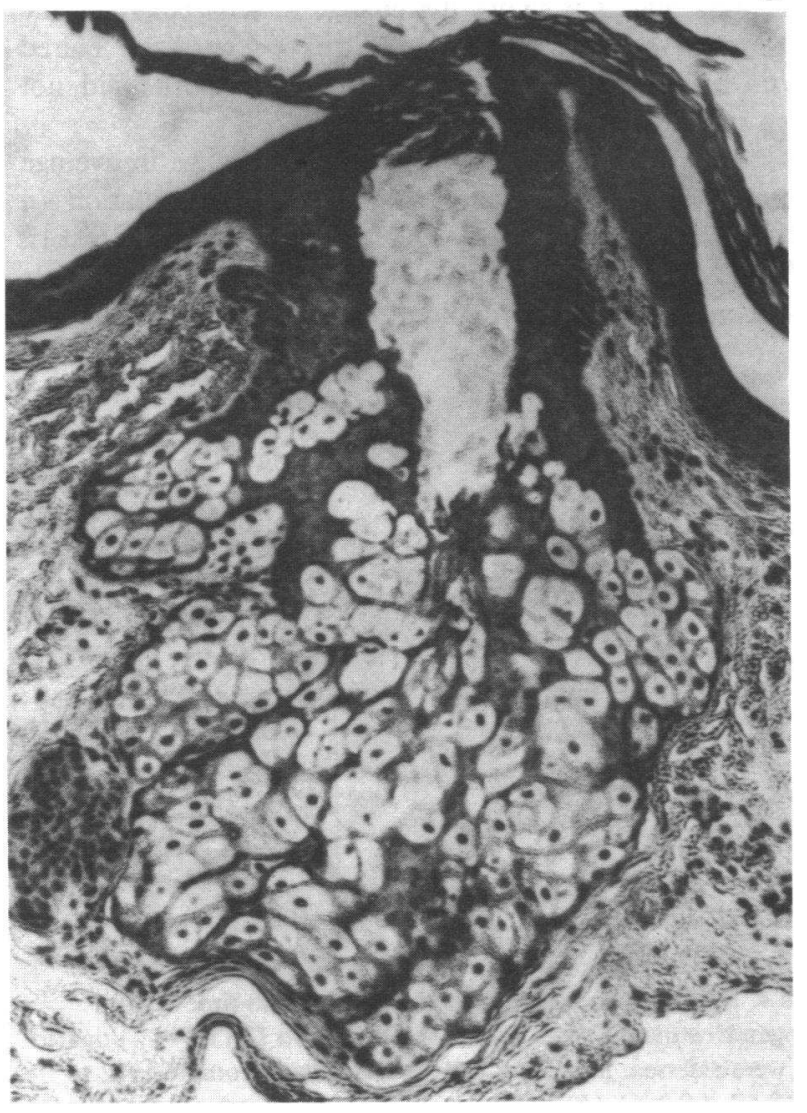

testosterone administration demonstrates the qualitative increase in sebaceous gland size. $\times 100$. 


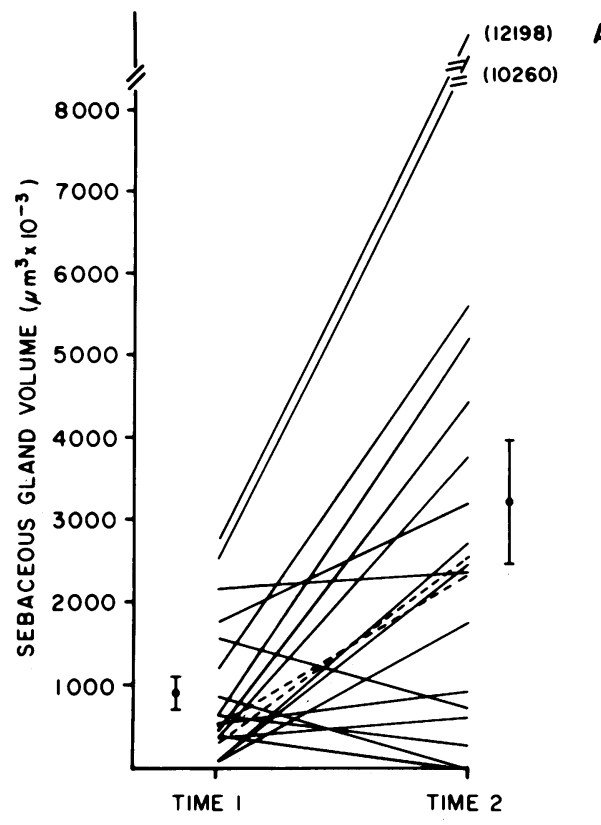

A

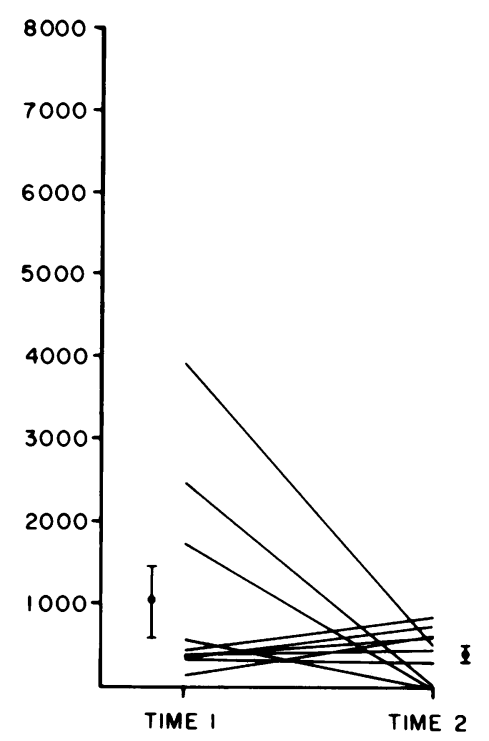

Figure 3. Human sebaceous gland volume before catheter implantation (time 1) and $26 \mathrm{~d}$ after implantation (time 2). (A) Androgen treatment group. $17 \mathrm{~T}$ treated $(\longrightarrow)$ and two DHT (- - -)-treated grafts were assessed. The mean sebaceous gland volume increased from $896 \pm 194$ to $3,233 \pm 754(P<0.001)$. (B) Control group. 10 nontreated grafts were compared at time 1 and time 2 . The mean sebaceous gland volume was $1,070 \pm 393$ at time 1 and $417 \pm 99$ at time 2 (NS) ( 1 mean \pm SEM).

biopsies, and the value was said to be zero for the sake of the calculations. Fig. 3 B shows the change in sebaceous volume after control catheter implantation in ten animals. Paired analysis revealed that the sebaceous gland volume did not change significantly in this group.

Sebaceous cell area. Fig. 4A shows the change in average sebaceous cell area pre- and post-androgen stimulation ( $n$ $=17)$. The average sebaceous cell area increased from $169 \pm 12$ $\left(\mu \mathrm{m}^{2}\right)$ to $243 \pm 19$ despite a smaller cell size in 4 of 17 grafts post-androgen treatment. A paired $t$ test evaluating the increase
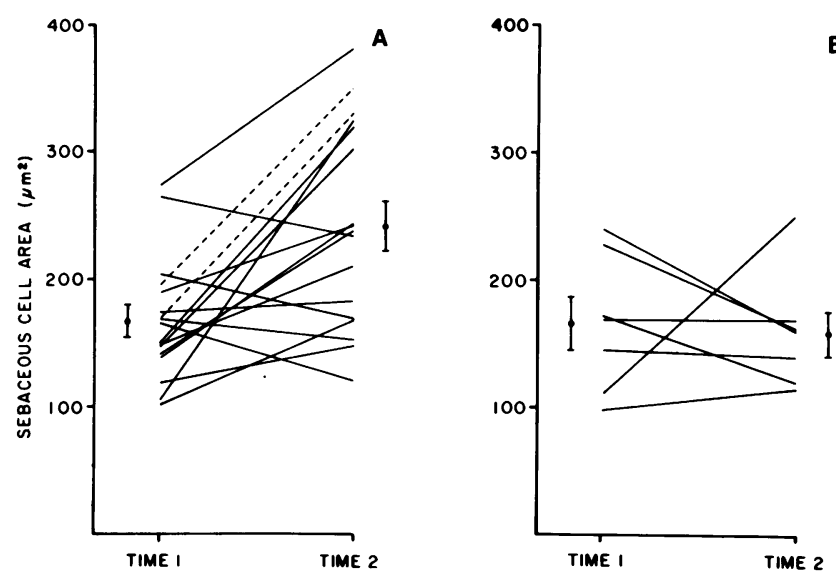

Figure 4. Human sebaceous cell area at time 1 and time 2. $(A)$ Androgen treatment group. $15 \mathrm{~T}(\longrightarrow)$ and two DHT (-- - -treated grafts were assessed. Sebaceous cell area increased from $167 \pm 12$ to $243 \pm 19(P<0.001)$. (B) Control group. Seven nontreated grafts were compared. Sebaceous cell area did not change significantly (\$ mean \pm SEM). in cell size was significant at the 0.001 level. Average sebaceous cell area in the control group $(n=7)$ was $168 \pm 17$ at time 1 and $153 \pm 16$ at time 2 (NS) (Fig. 4 B). Five grafts lacked an intact sebaceous gland with which to calculate sebaceous cell area.

Labeling index. Fig. 5 shows the effect of androgen stimulation on the labeling index on 12 mice. The labeling index (percentage) of the androgen-treated sebaceous glands increased from $2.7 \pm 0.7$ to $6.4 \pm 0.9$, which was significant at the 0.01 level using a paired $t$ test. In the control group, the labeling index was $5.1 \pm 1.9$ at time 1 and $3.2 \pm 1.1$ at time $2(n=4)$. The number of pairs was too small to test statistically.

Halowax experiment. The sample size in these experiments are small (10 Halowax treated and five vehicle-treated grafts). However, we felt that the results were worthy of presentation because they involved potential applications of this model,

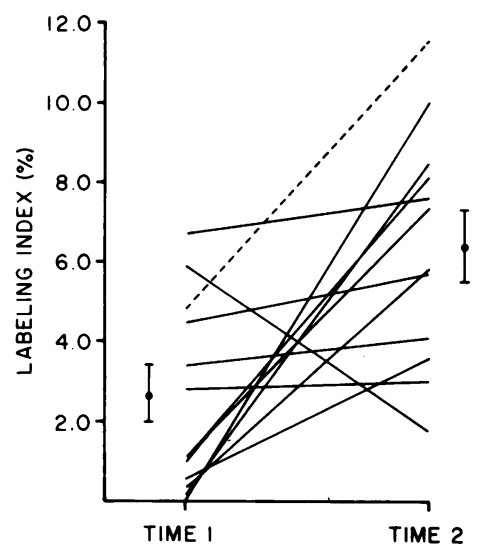

Figure 5. Labeling index of the human sebaceous glands in the androgen treatment group at time 1 and time 2. 11 T-treated mice $(-)$ and one DHTtreated (- - ) animal were available for analysis. The labeling index increased from $2.7 \pm 0.7$ to $6.4 \pm 0.9(P$ $<0.01)$ (\$ mean \pm SEM). 
which included the study of follicular dyskeratinization and the comedogenicity of topically applied substances. Fig. 6 is a photomicrograph of a microcomedo present after Halowax application. No visible comedones were produced. Before application of Halowax or vehicle, microcomedones were present in $70 \%$ of the Halowax and all of the vehicle-treated grafts. After application of Halowax or vehicle, microcomedones were seen in 14 of the 15 grafts. Twice as many microcomedones per graft were present in the Halowax group (5.2 \pm 1.0$)$ compared with the vehicle-treated grafts $(2.4 \pm 1.0)$. Androgen treatment did not appear to facilitate microcomedo formation.

\section{Discussion}

This study has developed an "animal" model to study the physiology and pathophysiology of human sebaceous glands. Essential to the model is the ability to successfully transplant human skin grafts that contain intact sebaceous glands onto the nude mouse. Comparative thickness grafts from human abdominoplasty skin will not successfully transplant to the nude mouse (G. G. Krueger). We hypothesize that this difference is related to the fact that the grafts used in this study were obtained from face skin. Skin graft survival is dependent on development of an adequate blood supply from the mouse within 3-5 d after grafting. We have shown that the graft vasculature, in part, retains human endothelial cells (G. G. Krueger, unpublished observations). This suggests that the degree of prior vascularity post-transplantation is dependent upon the degree of vascularity of the donor skin. The microvascular network of face skin is two to three times greater than other parts of the body, excepting palms and soles (22). Thus, grafts obtained from face skin would develop a larger capillary network post-grafting.

After successful transplantation of intact human sebaceous glands onto the nude mouse, we studied the response of these glands to administered androgens. In the majority of human studies, sebaceous gland response to androgens has been measured by changes in sebum production. Postulating that sebaceous gland size reflects sebum production, we elected to measure sebaceous gland volume. Data from the literature support this extrapolation. Strauss et al. (23) reported a marked increase in sebaceous gland size in pre-pubertal subjects after testosterone administration, although quantitative evaluation of sebaceous gland volume was not performed. Using planimetric measurements, Plewig (24) demonstrated an increased sebaceus gland volume in acne patients, compared with skin from normal persons.

The mean sebaceous gland volume in the androgen-treated skin increased $260 \%$, compared with no change in the control grafts. Sebaceous gland hyperplasia was seen as early as $18 \mathrm{~d}$ after catheter implantation. In addition to demonstrating a stimulatory effect of testosterone on the grafted sebaceous glands in 17 animals, we demonstrated a similar effect using DHT in two cases. To our knowledge, this is the first time that parenteral DHT has been shown to stimulate human sebaceous glands, although this effect has been well documented in rodents (2). The magnitude of increase of sebaceous gland volume was not the same for all grafts, and in four grafts the sebaceous gland volume decreased in the treatment group. In one graft, the lack of response was attendant with an unexpectedly low $\mathrm{T}$ level. The remaining three grafts were each from different donors. In each case, other grafts from the same donor demonstrated an increase in sebaceous gland volume in response to androgens. These data suggest that the apparent lack of response is not donor dependent. It is more likely that the lack of response represents intra-graft sampling variability of sebaceous glands. This variability is unavoidable and underscores the need for an adequate sample size.

Sebaceous cell area and the labeling index were also used to assay androgenic response. It is apparent from these data that the increase in sebaceous gland volume was due to increases in both sebaceous cell size and the number of sebaceous cells. More specifically, the percentage increase in the mean sebaceous gland area (before the volume conversion) in the treated grafts was three times the percentage increase in the sebaceous cell area. Thus, both mitotic and lipogenic activity were increased with testosterone and DHT stimulation. More extensive studies are needed to determine if there are differential actions of other androgens on the sebaceous gland, as has been proposed by some researchers (25). Again, the cell size in four grafts fell despite androgen stimulation. These were not the same grafts that demonstrated a decreased sebaceous gland volume, except in the case of the graft attendant with the low $\mathrm{T}$ level. We attribute this decrease in cell size to the intra-graft sampling variability.

Our autoradiographic technique utilized in vitro labeling to eliminate the possibility of residual radioactivity from previous in vivo labeling interfering with assessment of labeled cells in subsequent biopsies. In general, we found the labeling index to be the least sensitive test in ascertaining androgen stimulation. Technical difficulties, such as obtaining an adequate number of sebaceous glands in the $1-\mathrm{mm}$ wide section of radiolabelled tissue, contributed to this lack of sensitivity.

One difficulty experienced throughout the study was a relatively high mortality rate of grafted mice $(31 / 60,51.7 \%)$. $81 \%(25 / 31)$ of these mice were among the first five groups grafted. This dropout rate was primarily due to a higher rate of technical failures in the first five groups (15\%, compared with $0 \%$ in groups VI and VII), and a higher percentage of mice deaths during graft maturation in the first five groups (40\%, compared with $20 \%)$. The average lifespan of an athymic mouse in our colony is $120-180 \mathrm{~d}$. The mice in groups I-V were older $90+$ d) when grafted, which contributed to the higher mortality rate. Younger mice $(<90-d-o l d)$ were used for grafting in the last two groups with a lower mortality. It is conceivable that the difference in mortality between the donor groups biased the data to some degree. We consider this unlikely, since androgen response was not donor dependent. Mouse mortality did affect our ability to draw conclusions about the effect of endogenous testosterone on the human skin grafts, since only two intact mice survived in the control group. 


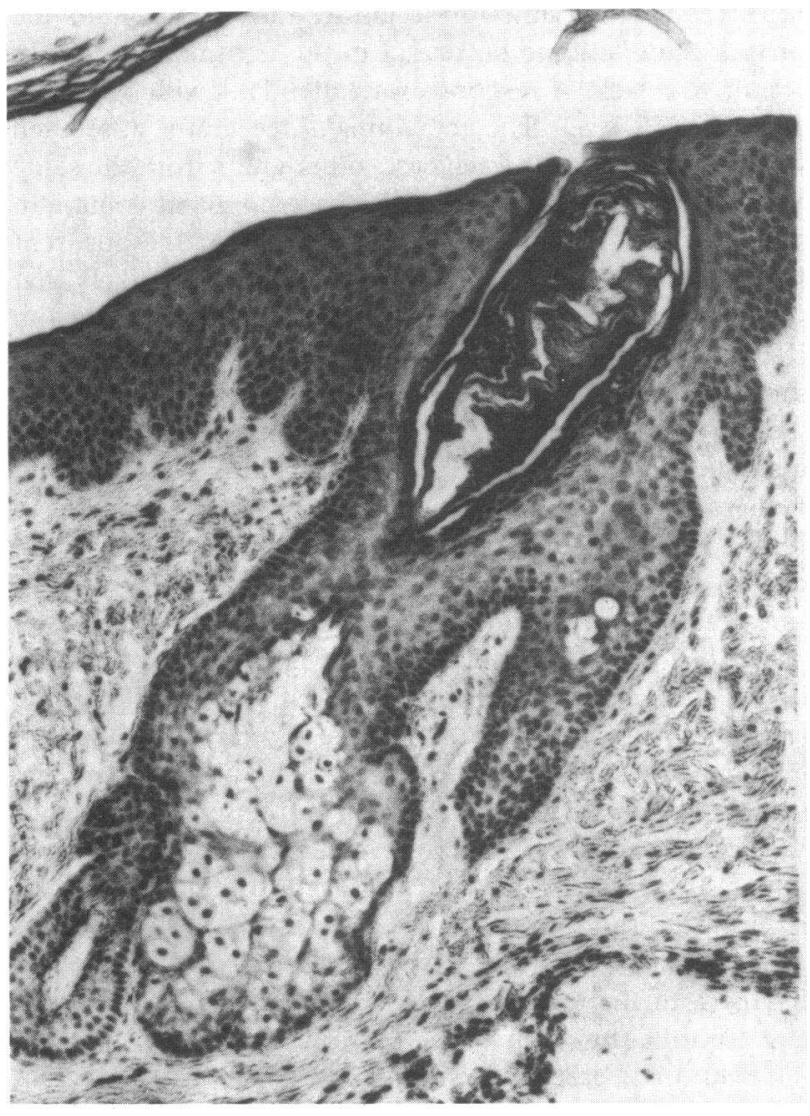

Figure 6. (A) Microcomedo from the human skin after Halowax application for $6 \mathrm{wk} . \times 100$. (B) A higher power of the same lesion

The attempt to produce chloracne in the skin grafts was somewhat less definitive. Microcomedones were definitely produced in greater numbers in the grafts that were treated with the chlorinated hydrocarbon, but no comedones were seen. Studies using Halowax 1014 (19) demonstrated comedo formation after 3-5 wk of application. Possible explanations for our inability to produce visible comedones include: an insufficient amount was applied; it needed to be applied under occlusion to keep the mice from removing it; the sebaceous follicles in the skin of older persons may be more resistant to known comedogens (26); and Halowax N-34 may be less comedogenic than Halowax 1014.

In 1979, research needs in acne investigation were outlined (27). Included were: $(a)$ development of human and animal models to investigate the sebaceous follicle, including the induction of follicular dyskeratoses by chemical and physical factors; $(b)$ further studies characterizing steroid receptors and cellular kinetics; $(c)$ characterization of metabolic pathways within the sebaceous gland, follicular epithelium, and surrounding dermis; $(d)$ investigation of the interrelated effects of steroidal and nonsteroidal hormones; $(e)$ study of the effect of

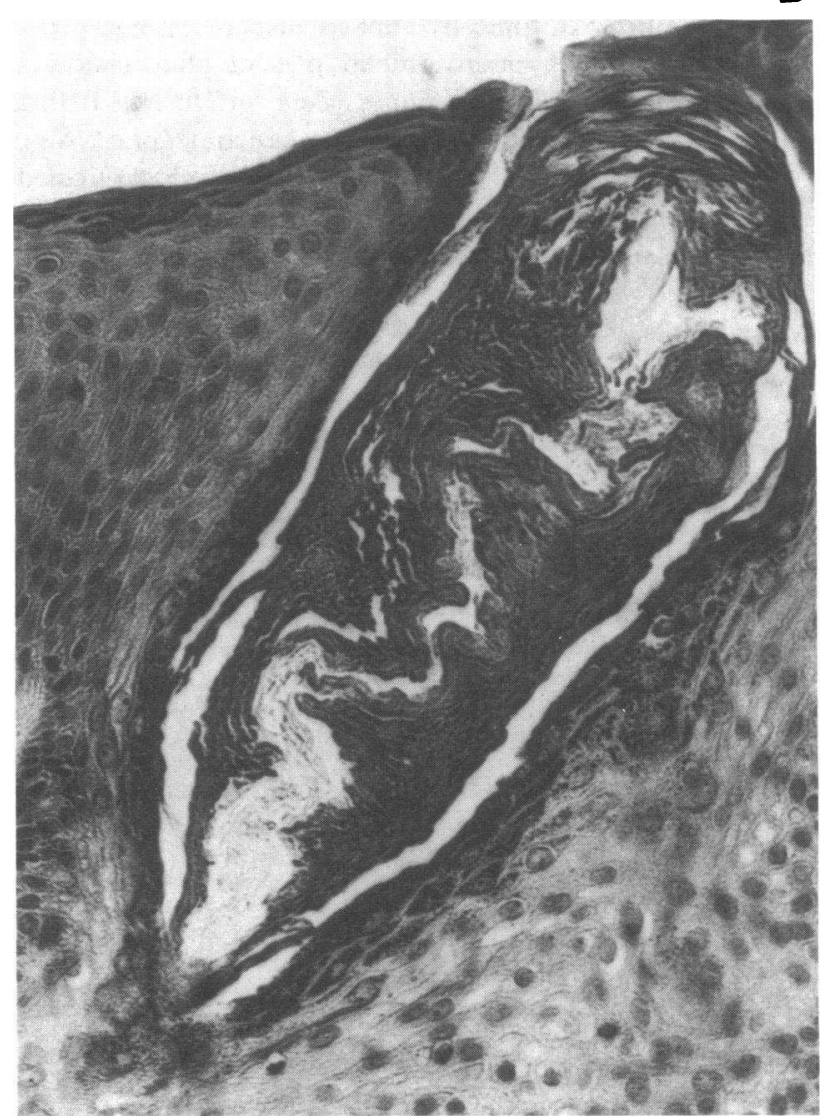

showing the compaction of the stratum corneum and the hypergranularity of the infra-infundibular epithelium. $\times 250$.

anti-androgens; and $(f)$ further study of lipid, prostaglandin, and arachidonic acid synthesis pathways in the sebaceous gland. Our model has potential to address all of the above research needs. The fact that it contains intact, functioning human sebaceous glands on an animal system allows the study of human sebaceous gland under controlled circumstances, which is not easily attained in situ.

In summary, this study demonstrates that: full thickness human face skin, which contains intact sebaceous glands, can be grafted and maintained on the nude mouse; grafted human sebaceous glands become hyperplastic in response to androgen administration; and microcomedones can be produced in the human skin grafts. This model has the potential to be a valuable research tool in the investigation of the physiology and pathology of sebaceous glands and sebaceous follicles.

\section{Acknowledgments}

This research was supported in part by National Institutes of Health grants AM21405, AM28412, and Hoffmann-La Roche, Inc., Nutley, NJ. 


\section{References}

1. Ebling, F. J. 1974. Hormonal control and methods of measuring sebaceous gland activity. J. Invest. Dermatol. 62:161-174.

2. Ebling, F. J., E. Ebling, V. McCaffery, and J. Skinner. 1971. The response of the sebaceous glands of the hypophysectomizedcastrated male rat to $5 \alpha$-dihydrotestosterone, androstenedione, dehydroepiandrosterone, and androsterone. J. Endocrinol. 51:181-190.

3. Thody, A. J., and S. Shuster. 1971. Effect of adrenalectomy and adrenocorticotrophic hormone on sebum secretion in the rat. $J$. Endocrinol. 49:325-328.

4. Ebling, F. J., E. Ebling, V. Randall, and J. Skinner. 1975. The sebotrophic action of growth hormone (BGH) on the rat. Br. J. Dermatol. 92:325-332.

5. Haskin, D., N. Lasher, and S. Rothman. 1953. Some effects of ACTH, cortisone, progesterone and testosterone on sebaceous glands in the white rat. J. Invest. Dermatol. 20:207-212.

6. Strauss, J. S., and A. M. Kligman. 1961. The effect of progesterone and progesterone-like compounds on the human sebaceous gland. $J$. Invest. Dermatol. 36:309-319.

7. Adachi, K. 1974. Receptor proteins for androgen in hamster sebaceous glands. J. Invest. Dermatol. 62:217-223.

8. Kligman, A. M., and T. Kwong. 1979. An improved rabbit ear model for assessing comedogenic substances. Br. J. Dermatol. 100:699702.

9. Hambrick, Jr., G. W., and H. Blank. 1956. A microanatomical study of the response of the pilosebaceous apparatus of the rabbit's ear canal. J. Invest. Dermatol. 26:185-200.

10. Kligman, A. M., and O. H. Mills, Jr. 1972. "Acne cosmetica". Arch. Dermatol. 106:843-850.

11. Rygaard, J. 1974. Skin grafts in nude mice. 3. Fate of grafts from man and donors of other taxonomic classes. Acta Pathol. Microbiol. Scand. Sect. A Pathol. 82:105-112.

12. Krueger, G. G., and R. A. Briggaman. 1982. The nude mouse in the biology and pathology of skin. In The Nude Mouse in Experimental and Clinical Research. J. Fogh and B. C. Giovanella, editors Academic Press, Inc., New York. 2:301-322.

13. Krueger, G. G., D. A. Chambers, and J. Shelby. 1980. Epidermal proliferation of nude mouse skin, pig skin and pig skin grafts: failure of nude mouse skin to respond to the tumor promoter 12-0-Tetradecahoyl Phorbol 13-Acetate. J. Exp. Med. 152:1329-1339.

14. Billingham, R. E., and P. B. Medawar. 1951. The technique of free skin grafting in mammals. J. Exp. Biol. 28:385-402.
15. DaMassa, D. A., D. Kobashigawa, E. R. Smith, and J. M Davidson. 1976. Negative feedback control of $\mathrm{LH}$ by testosterone: a quantitative study in male rats. Endocrinology. 99:736-742.

16. Meikle, A. W., J. D. Stringham, D. E. Wilson, and L. I. Dolman. 1979. Plasma $5 \alpha$-reduced androgens in men and hirsute women: role of adrenals and gonads. J. Clin. Endocrinol. Metab. 48:969-975.

17. Picton, W., H. DeVitt, and M.A. Forgie. 1976. Practical applications of the Quantimet 720, an image analysing computer, in the field of investigative dermatology. Br. J. Dermatol. 95:341-348.

18. Ebling, F. J. 1957. The action of testosterone on the sebaceous glands and epidermis in castrated and hypophysectomized male rats. J. Endocrinol. 15:297-306.

19. Hambrick, Jr., G. W. 1957. The effect of substituted naphthalenes on the pilosebaceous apparatus of rabbit and man. J. Invest. Dermatol. 28:89-102.

20. Puhvel, S. M., M. Sakamoto, D. C. Ertl, and R. M. Reisner. 1982. Hairless mice as models for chloracne: a study of cutaneous changes induced by topical application of established chloracnegens. Toxicol. Appl. Pharmacol. 64:492-503.

21. Colton, T. 1974. Statistics in Medicine. Little, Brown \& Co., Boston. 134-136, 219-221.

22. Ryan, T. J. 1973. Structure, pattern and shape of the blood vessels of the skin. In The Physiology and Pathophysiology of the Skin. Vol. 2. A. Jarrett, editor. Academic Press Inc., London. 577649.

23. Strauss, J. S., A. M. Kligman, and P. E. Pochi. 1962. The effect of androgens and estrogens on human sebaceous glands. $J$. Invest. Dermatol. 39:139-155.

24. Plewig, G. 1974. Acne vulgaris: proliferative cells in sebaceous glands. Br. J. Dermatol. 90:623-630.

25. Ebling, F. J., E. Ebling, V. McCaffery, and J. Skinner. 1973. The responses of the sebaceous glands of the hypophysectomizedcastrated male rat to $5 \alpha$-androstanedione and $5 \alpha$-androstane- $3 \beta, 17 \beta$ diol. J. Invest. Dermatol. 60:183-187.

26. Cunliffe, W. J., and J. A. Cotterill. 1975. Histology. In The Acnes, Clinical Features, Pathogenesis and Treatment. A. Rook, editor. W. B. Saunders Co. Ltd., London. 62-95.

27. Shalita, A., R. K. Freinkel, D. Downing, A. Kligman, J. J. Leyden, W. Montagna, P. Pochi, S. M. Puhvel, R. M. Reisner, J. S Strauss, and G. Sansone-Bazzano. 1979. Analysis of research needs and priorities in dermatology. J. Invest. Dermatol. 73:434-442. 